\title{
Bilateral procedures in randomised controlled trials
}

A number of specific statistical problems arise in the handling of data from particular branches of medicine. In orthopaedics a perennial question concerns the use of data from patients on whom operations have been performed on both sides of the body. For many years, the results of series of joint replacements have been reported in terms of the number of joints operated on rather than the number of patients. If a patient had both left and right hips replaced the authors usually regarded the outcome data as two distinct observations. This may, however, be an unsound practice, since we cannot be sure that the two outcomes are strictly independent of each other. For instance: if one replaced hip does not perform well, the patient may compensate by putting more weight on the other; the patient's lifestyle (whether active or sedentary) and any metabolic or genetic factors which may influence outcome will affect both hips; and when asked for an opinion, the patient's tendency to be optimistic (or pessimistic) will be reflected in two outcomes not one. To regard the outcomes of replacement of both hips in one patient as if they were two patients each with a single replacement needs therefore to be justified.

The problem has been illustrated by Donner and Donald (1988) in a dental example in which the difference in incidence of dental caries between men and women was investigated. A cohort of patients with exposed roots had various numbers of tooth surfaces at risk: 40 patients contributed 126 surfaces. When all the surfaces were regarded as statistically independent, the difference between the sexes was highly significant $(p=0.01)$, but when the similarity of outcome between different surfaces in the same patient was allowed for, the analysis gave a $\mathrm{p}$ value of only 0.07 .

The issue becomes important when a randomised trial is being reported, as in the paper on metatarsal osteotomies by Klosok et al in this issue (p. 825). Whenever formal statistical significance tests are used, the notion of independent observations becomes critical. Klosok et al report outcomes in terms of continuous variables (valgus angles, metatarsal lengths) and categorical variables (presence or absence of callosities or metatarsalgia) and different strategies are required for each. The following suggestions are made for authors whose data refer to bilateral and unilateral procedures.

R. W. Morris, MSc, Senior Lecturer in Medical Statistics Department of Public Health and Primary Care, Royal Free Hospital School of Medicine, Rowland Hill Street, London NW3 2PF, UK.

C) 1993 British Editorial Society of Bone and Joint Surgery 0301-620X/93/5660\$2.00

J Bone Joint Surg [Br] 1993; 75-B :675-6.
Random allocation. Randomise the patients rather than the limbs. When two treatments are compared patients with bilateral disease should receive the same treatment on both sides.

Number of units of analysis. In the study referred to above, 26 patients underwent the Wilson osteotomy and 25 the chevron osteotomy. Although 87 operations were performed (42 of one type of osteotomy and 45 of the other), the total sample size is 51 , not 87 , and the significance tests will then be appropriately conservative. Management of continuous variables. In bilaterally operated patients the range of motion, for example of the big toe, was taken to be the average for the two feet. Unilaterally and bilaterally operated patients then had one value each, and the means for the two groups could be compared using one-way analysis of variance. This approach, which was recommended by Rosner (1982), may be criticised as underweighting the data from patients with bilateral disease, and this objection would have force if the results from the two feet of the same patient tended to differ widely. Differential weighting, however, of unilateral and bilateral observations is complex and depends on the relative size of the betweensubject and within-subject variations. The greater the within-subject variation, the more weight should be given to bilateral patients. Armitage and Berry (1987) provide details of the analysis of such data once the weights have been decided upon. Fortunately, this sophistication is usually unnecessary in a randomised trial since the proportion of bilateral patients is similar in the two groups.

Management of categorical variables. Klosok et al sought evidence of callosity in both feet of the bilaterally operated patients, and recorded it as present if it was found in at least one foot. A plausible objection to this method is that bilaterally operated patients may be more likely to develop callosities than unilaterally operated patients. Again, this does not affect the results if bilaterally operated patients are equally distributed between the two treatment groups. If they are not, stratified analysis can solve the problem, the appropriate technique being the Mantel-Haenszel modification of the chi-squared test (Armitage and Berry 1987).

Survival analysis. One issue not considered in the review by Carr et al (1993) of survival analysis of joint replacements is how to handle bilateral procedures which, unless they are carried out simultaneously, are not even followed up for the same length of time. Furthermore, one joint may have been replaced with Mark I of the prosthesis and the other with Mark II. The ingenious analyses of Rosner (1982) and Donner and Donald (1988) both assume that each of several observations made on a particular patient results from the same treatment. 
One solution, in randomised trials, would be to use data only from the first of the two joints replaced, but this approach may seem too conservative, given the very low failure rates reported in most series. It may well be that the 'dependency effect' is relatively unimportant. My own analysis of a series of knee replacements failed to show dependency between results of left and right knees among 98 patients operated upon bilaterally, since there were no patients in whom failure occurred in both knees. It did appear, however, that patients with bilateral replacements had lower failure rates than the rest and this was especially the case for the second of two replaced knees. If this result, which may be an effect of patient selection or of reduced functional demand, can be generalised, then failure rates reported in case series with a high proportion of bilateral replacements will tend towards undue optimism.

Conclusions. In the analysis of the results of orthopaedic procedures the patient should be regarded as the unit of observation rather than the procedure. When bilateral procedures are included, the results should be condensed where possible into a single observation; otherwise the first procedure only should be included in the data. Differential weighting is unnecessary in randomised trials if the proportion of bilateral procedures is similar in the two treatment groups. If the proportions differ, stratified analysis is recommended.

RICHARD W. MORRIS

\section{REFERENCES}

Armitage P, Berry G. Statistical methods in medical research. Second ed. Oxford: Blackwell Scientific, 1987.

Carr AJ, Morris RW, Murray DW, Pynsent PB. Survival analysis in joint replacement surgery. J Bone Joint Surg $[\mathrm{Br}]$ 1993; 75-B: 178-82.

Donner A, Donald A. The statistical analysis of multiple binary measurements. J Clin Epidemiol 1988; 41 :899-905.

Rosner B. Statistical methods in ophthalmology: an adjustment for the intraclass correlation between eyes. Biometrics 1982; 38:105-14. 